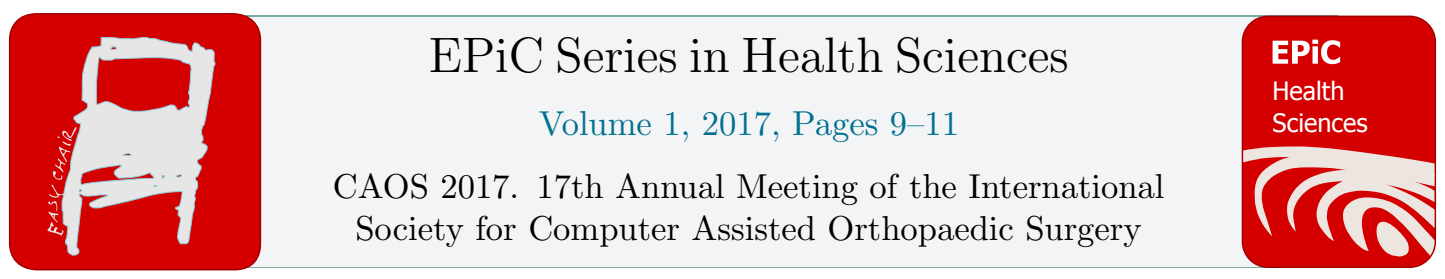

\title{
Non-invasive navigated assessment of the lower limb axis prior to total knee arthroplasty. Comparison with conventional navigated assessment.
}

\author{
Jean-Yves Jenny, Gauthier Dillmann, Vincent Gisonni, Henri Favreau \\ University Hospital Strasbourg, Strasbourg, France \\ jean-yves.jenny@chru-strasbourg.fr
}

\begin{abstract}
The non-invasive navigation system may help the surgeon to assess the lower limb axis at least as accurately as with the conventional radiographic techniques without exposure. But validation of this system used was only performed experimentally, and the present study is the first step of clinical validation.
\end{abstract}

\section{Introduction}

Assessment of the lower limb axis is a routine procedure for several orthopedic procedures used to address knee osteoarthritis. There is unfortunately no accepted gold standard for this measurement. Assessment on long leg X-rays is recommended in clinical practice (Holme 2015), but it involves several biases, especially because of the inconsistent flexion and rotational positioning of the leg when X-rays are taken (Khare 2016). Navigated techniques are considered to be accurate and precise (Jenny 2004, Chauhan 2004), but most of the systems used involve direct fixation of arrays to the bone, making this technique invasive and dedicated to operating room only.

A non-invasive navigated (NIN) technique has been developed to assess the lower limb axis, replacing the conventional invasive array fixation by a non-invasive one (Clarke 2012). The goal of the device is to allow measuring the lower limb axis in a standing position with full weight bearing without radiation exposure with an easy and fast process. The purpose of the current study was to assess the accuracy and precision of this technique by comparison to the conventional, invasive navigated (IN) measurement technique. The tested hypothesis was that NIN measure of the lower limb axes will significantly differ from IN measure when performed on a supine patient under general anesthesia just before total knee Arthroplasty (TKA). The secondary goal was to assess the intra- and inter-observer reproducibility of the NIN system. 


\section{Material and methods}

All patients scheduled for a TKA from May 2015 to June 2015 were eligible for the study. Inclusion criteria were: an end-stage knee osteoarthritis scheduled for primary unilateral or bilateral TKA, intra-operative assistance of the navigation system routinely used at the institution during TKA, availability of the non-invasive navigated system, availability of the resident performing the study. Exclusion criteria were: a previous surgery on the same knee (excepting knee arthroscopy), a revision TKA. NIN and IN measures were performed sequentially on a supine patient after general anesthesia. Following data were recorded: coronal mechanical femoro-tibial angle at maximal extension angle without stress and coronal mechanical femoro-tibial angle at $30^{\circ}$ of knee flexion without stress.

Comparison between NIN and IN measures was performed with a paired Wilcoxon test. The mean paired difference was calculated; the difference was considered as an outlier if it was $> \pm 3$ degrees, and the equivalence test was performed. Correlation between NIN and IN measures was assessed by calculation of the Spearman correlation test. Agreement between NIN and IN measures was assessed by the graphic Bland-Altman method with definition of the systematic bias and subsequent correction of the paired differences. Repeatability was assessed by calculation of the intra-class correlation coefficient (ICC) and its $95 \%$ confidence interval (CI). $\mathrm{P}<0.05$ was considered significant.

\section{Results}

There was a significant difference between NIN and IN measures at maximal extension $(p=0.01)$ and at $30^{\circ}$ of flexion $(\mathrm{p}=0.02)$. There was a good correlation between NIN and IN measures at maximal extension (rho $=0.80, \mathrm{p}<0.05)$ and a weak correlation between NIN and IN measures at $30^{\circ}$ of flexion (rho $=0.43, \mathrm{p}<0.05$ ). There was a good agreement between NIN and IN measures at maximal extension ( $\mathrm{rho}=0.13, \mathrm{p}=0.43$ ) with a systematic bias of +1.6 degrees. There was a good agreement between NIN and IN measures at $30^{\circ}$ of flexion ( $(\mathrm{hho}=0.10, \mathrm{p}=0.56$ ) with a systematic bias of +3.1 degrees. There was an excellent repeatability between NIN and IN measures at maximal extension (ICC $=0.88, \mathrm{CI} 0.71-0.95$ ), and a poor repeatability between NIN and IN measures at $30^{\circ}$ of flexion (ICC $=0.52$, CI $0.11-0.78$ ). After correction of the systematic bias, the differences were not significant and only 5 differences at maximal extension (25\%) and 11 measures at $30^{\circ}$ of flexion $(55 \%)$ were considered as outliers.

For the first observer, there was an excellent repeatability between the two measures at maximal extension (ICC $=0.70$, CI $0.29-0.89$ ), and a poor repeatability between the two measures at $30^{\circ}$ of flexion (ICC $=0.49$, CI $0.00-0.80$ ). For the second observer, there was a good repeatability between the two measures at maximal extension ( ICC $=0.73$, CI 0.34-0.90), and a good repeatability between the two measures at $30^{\circ}$ of flexion (ICC $=0.66$, CI $0.23-0.88$ ). Inter-observer repeatability was excellent for measures at maximal extension (ICC $=0.84, \mathrm{CI} 0.58-0.95$ ), and poor for measures at $30^{\circ}$ of flexion ( $\mathrm{ICC}=0.55$, CI 0.05-0.83).

\section{Discussion}

The main conclusion of the accuracy study was that the null hypothesis was rejected. NIN measures differed significantly from IN measures for all three items analyzed. These differences may occur because of skin motion artifact. The non rigid fixation of the NIN trackers on the skin instead of being secured to the bone may induce uncontrolled displacement of the marker with respect to the bone itself (Benoît 2015, Sangeux 2006). Russell (Russell 2014) observed a variation of $3^{\circ}$ between 
NIN and IN measures of the coronal mechanical femoro-tibial axis with the same hardware and softwares, which is consistent with the present results. Another confounding factor may be the difference between the two algorithms for NIN or IN calculation.

Despite these limitations, the present study may induce some relevant improvements in the clinical practice. There is a definite need for accurate and precise measures of leg alignment for arthritic patients, especially when a TKA is considered. The appropriate type of implant and the need for ligamentous releases may be detected prior to surgery when analyzing deformities. NIN systems may help the surgeon to address these points at least as accurately as with the conventional radiographic techniques without exposure. But validation of the NIN system used was only performed experimentally, and the present study is the first step of clinical validation.

\section{References}

1. Benoit DL, Damsgaard M, Andersen MS, Surface marker cluster translation, rotation, scaling and deformation: Their contribution to soft tissue artefact and impact on knee joint kinematics, J Biomech, 48(10), pp: 2124-2129, 2015.

2. Chauhan SK, Clark GW, Lloyd S, Scott RG, Breidahl W, Sikorski JM, Computer-assisted total knee replacement. A controlled cadaver study using a multi-parameter quantitative CT assessment of alignment (the Perth CT Protocol), J Bone Joint Surg Br, 86(6), pp: 818-823, 2004.

3. Clarke JV, Riches PE, Picard F, Deakin AH, Non-invasive computer-assisted measurement of knee alignment, Comput Aided Surg, 17(1), pp: 29-39, 2012.

4. Holme TJ, Henckel J, Hartshorn K, Cobb JP, Hart AJ, Computed tomography scanogram compared to long leg radiograph for determining axial knee alignment, Acta Orthop, 86(4), pp: 440-443, 2015.

5. Jenny JY, Boeri C, Picard F, Leitner F, Reproducibility of intra-operative measurement of the mechanical axes of the lower limb during total knee replacement with a non-image-based navigation system. Comput Aided Surg, 9(4), pp: 161-165, 2016.

6. Khare R, Jaramaz B, Accuracy of leg alignment measurements from antero-posterior radiographs, Biomed Tech (Berl), Aug 29, 2016. pii: /j/bmte.ahead-of-print/bmt-2015-0221/bmt-2015221.xml, doi: 10.1515/bmt-2015-0221, [Epub ahead of print].

7. Russell DF, Deakin AH, Fogg QA, Picard F, Quantitative measurement of lower limb mechanical alignment and coronal knee laxity in early flexion, Knee, 21(6), pp:1063-1068, 2014.

8. Sangeux M, Marin F, Charleux F, Dürselen L, Ho Ba Tho MC, Quantification of the 3D relative movement of external marker sets vs. bones based on magnetic resonance imaging, Clin Biomech, 21(9), pp: 984-991, 2006.

\section{Disclosure}

Jean-Yves JENNY receives royalties from Aesculap, is a paid consultant for Exactech, is an unpaid consultant for FH Orthopedics, is member of the board of the CAOS-International Society and of the French Society for Hip and Knee Surgery 